\title{
Cigarette smoking and risk of disabling dementia in a Japanese rural community: a nested case-control study
}

Ai Ikeda ${ }^{1}$, Kazumasa Yamagishi ${ }^{2}$, Takeshi Tanigawa ${ }^{2}$, Renzhe Cui ${ }^{2}$, Masayuki Yao ${ }^{3}$, Hiroyuki Noda ${ }^{1}$, Mitsumasa Umesawa ${ }^{2}$, Choy-Lye Chei ${ }^{2}$, Kimiko Yokota ${ }^{2}$, Yumi Shiina ${ }^{4}$, Mitiko Harada ${ }^{4}$, Keiko Murata ${ }^{5}$, Takashi Asada ${ }^{6}$, Takashi Shimamoto ${ }^{7}$, Hiroyasu Iso ${ }^{1}$

1. Public Health, Department of Social and Environmental Medicine, Graduate School of Medicine, Osaka University, Osaka, Japan.

2. Department of Public Health Medicine, Doctoral Program in Social and Environmental Medicine, Graduate School of Comprehensive Human Sciences, University of Tsukuba, Ibaraki, Japan.

3. Department of Psychiatry, Ranryoen Hospital, Osaka, Japan.

4. Kyowa Public Health Center, Chikusei Municipal Office, Ibaraki, Japan.

5. Tone Public Health Center, Tone Municipal Office, Ibaraki, Japan.

6. Department of Clinical Neuroscience, Doctoral Program in Medical Science for Control of Pathological Process, Graduate School of Comprehensive Human Sciences, University of Tsukuba, Ibaraki, Japan.

7. Osaka Medical Center for health Science and Promotion, Osaka, Japan.

Corresponding author:

Prof. Hiroyasu Iso

Public Health, Department of Social and Environmental Medicine, Graduate School of Medicine, Osaka University, Yamadaoka 2-2, Suita, Osaka 565-0871, Japan

Phone: +81-6-6879-3911 Fax: +81-6-6879-3919 Email Address:fvgh5640@mb.infoweb.ne.jp

Short title: Cigarette smoking and risk of disabling dementia

Key words: Dementia, smoking, stroke 


\section{ABSTRACT}

Background: Previous prospective cohort studies have examined the association between smoking and the risk of dementia, but the results were inconsistent. Methods: A prospective, nested, case-control study was conducted to examine the association between cigarette smoking and risk of disabling dementia within the cohort of 6,343 men and women aged 35-85 years. Incident dementia was documented in 208 men and women (95 cases with and 113 cases without history of stroke). Two control subjects per case were selected by matching for sex, age and year of examination. Results: The multivariable odds ratios (95\% CI) for current versus never-smokers were 2.3 (1.1-4.7) for total dementia, 2.6 (0.8-8.2) for dementia with history of stroke and 2.2 (0.8-5.7) for dementia without it, yielding no effect of stroke history on the smoking-dementia association. A dose-response relationship was noted between the years of cigarettes smoking and the risk of total dementia and a significant excess risk was found for smokers of $\geq 45$ years in duration. Conclusions: The present prospective study suggests that a long-term cigarettes smoking may raise the risk of disabling dementia. 


\section{Background}

Dementia has become a major health problem in Japan, as well as in Western countries, because of the sharp increase in the number of elderly individuals. ${ }^{1}$ Stroke produces decline in cognitive functions and dementia ${ }^{2}$, and the traditional risk factors for cardiovascular disease, such as hypertension ${ }^{2-5}$, high cholesterol ${ }^{3,5}$, and diabetes ${ }^{2,5}$ are associated with increased risks for both Alzheimer's disease and vascular dementia. By contrast, the contribution of smoking, major risk factor for cardiovascular disease, to dementia is less clear. Several previous case-control studies have indicated that smoking is associated with a reduced risk of dementia. ${ }^{6}$ However, this inverse association between smoking and dementia might have been caused by the introduction of differential survival biases. ${ }^{6,7}$ Smokers are likely to have been under-represented in these studies because of their increased risk of early death from cardiovascular disease and cancer. ${ }^{8}$ In addition, individuals with dementia might have tended to under-report their smoking habits. ${ }^{6,7}$ These biases could have distorted the reported associations between smoking and dementia. ${ }^{6,7}$ Thus, owing to the methodological limitations of case-control studies, there is a requirement for prospective studies to examine the association between smoking and dementia.

Some recent prospective cohort studies have suggested that smokers have an approximately 1.4-fold to 1.7-fold increased risk of dementia compared with individuals who have never smoked, ${ }^{6,9-11}$ although other studies of this type have shown no such association. ${ }^{6}$ The aim of the present nested case-control study was to investigate the association between cigarette smoking and the risk of disabling dementia using prospective data from members of a Japanese rural community. 


\section{MATERIALS AND METHODS}

The study cohort was established in the town of Kyowa (Ibaraki Prefecture, Japan) in 1981 for community-based cardiovascular prevention program. The design of the cohort study has been described previously. ${ }^{12,13}$ This cohort has been followed up with annual cardiovascular surveys, and surveillance for incidence and mortality of stroke and coronary heart disease. Within this cohort, elderly persons aged $\geq 65$ years with disabling dementia, requiring for care were identified under the national long-term care insurance program, between April 1, 2000 and March 31, 2004. We did not have information on cognitive function prior to dementia. However, in order to reduce the potential effects of as yet undiagnosed dementia at the time of a given cardiovascular survey, the cohort included 6,343 men and women aged 35-85 years who participated in the surveys between 1981 and 1994 (of these, 4,085 subjects were over 65 years old between 2000 and 2004). Of these, 1,156 subjects were died, 252 subjects were moved out of study area before March 31, 2004. The overall participation rate was $70 \%$. The Ethics Committee of the University of Tsukuba, Japan, approved the study procedures.

\section{Long-term care insurance program for elderly aged $\geq 65$}

The details of the national long-term care insurance program for elderly aged $\geq 65$ have been

reported elsewhere. ${ }^{14,15}$ In brief, the insurance program was started in Japan from April 2000. This program was essentially extended from the national health insurance system, and partially relied on subsides from general revenues from national, prefectures and municipalities. All individuals aged $\geq 40$ are required to pay a supplement to their health insurance, which is transferred to the long-term care. The payment is directly withdrawn from their monthly income, shared with the employer or deducted from their public pensions. All 
individuals are able to receive a long-term care through their resided municipals when they become 65 and older and also have disabling dementia and/or reduced activity of daily living.

\section{Case-control analysis}

\section{Case selection}

The cases were selected among the subjects aged >65 years between 2000 and 2004 who were regarded as suffering from dementia under the long-term insurance program. The dementia status were classified into six-ranks $(0-\mathrm{V})$ and were reported by their primary care physicians according to the standardized physicians’ manual issued by the Health and Welfare Bureau for the Elderly of Japan. ${ }^{16}$ The dementia status were annually updated and were reviewed until they were withdrawn due to death or moving out of the study area. Individuals without dementia were classified as rank 0. Individuals who were diagnosed with mild cognitive dysfunction, but who had no dementia-related symptoms or behavioural disturbance and were capable of living independently, were classified as rank I . Individuals who had moderate dementia-related behavioural disturbance and cognitive impairment with slight dependence were classified as rank II . Individuals who had moderate-to-severe dementia-related behavioural disturbance and cognitive impairment with moderate dependence were classified as rank III. Individuals who had severe dementia-related behavioural disturbance and cognitive impairment with heavy dependence were classified as rankIV. Finally, individuals with severe dementia-related behavioural disturbance and cognitive impairment who required medical treatment were classified as rank V . Individuals who were ranked as $\mathbb{I}$ or greater for the first time were regarded as cases in the present analysis. The validation for the cut-off point was examined by neuropsychiatrists through the subjects' cognitive functions (attention, memory, visuospatial function, language and reasoning) based on their ageing-associated cognitive decline (AACD), as defined by the International Psychogeriatric Association. ${ }^{17}$ The 
calculated sensitivity and specificity values from the validation study of 34 subjects were 36\% and $90 \%$, respectively.

\section{History of stroke identification}

The history of stroke was obtained from the annual cardiovascular surveys, surveillance for cardiovascular disease ${ }^{13}$ from 1981 to the present, and/or statements from the primary care physicians. In present study, 85\% of the stroke history was confirmed based on CT or MRI using standardized criteria. ${ }^{18}$ The determination of stroke history without the imaging studies was conducted based on the clinical criteria. ${ }^{19}$ Stroke was defined as rapid onset focal neurological disorder persisting for $\geq 24 \mathrm{~h}$, or until death. Transient ischemic attack was not included.

\section{Control selection}

We employed the risk-set sampling ${ }^{20}$ of controls, i.e., matching randomly each case of dementia with two of all other individuals with no diagnosis of dementia in the study cohort who were alive and resided within the community on the date of diagnosis of dementia for the case and who had the same gender, year of given annual cardiovascular survey and age $( \pm 2$ years) as the case. The vital status of controls was assessed before the control selection.

\section{Measurement of risk factors}

Participants were interviewed smoking status (current, past, or never); those who smoked cigarettes were asked their age at start of smoking and the average daily number of cigarettes smoked at the time of a given annual cardiovascular survey. Those who had not smoked for 3 months or more were defined as past smokers. The past smokers were asked for their age at starting and quitting smoking, and the average daily number of cigarettes smoked. For each case, the latest measurements from a given survey between 1981 and 1994 were selected for the analysis. For controls, the measurements included in the analysis were selected from the same year of a given survey as the case. 
The reliability of smoking questionnaire was tested in the same population. The proportion of upholding the same smoking status at 1 year after the time of a given survey between 1981 and 1994 ( $n=2314)$ was 97\% for never-smokers, 87\% for past smokers, 93\% for current smokers ( $83 \%$ for smokers of $<20$ cigarettes per day, and $75 \%$ for smokers of $>20$ cigarettes per day).

Confounding variables included systolic blood pressure (mmHg), use of antihypertensive medication (yes and no), body mass index (BMI) $\left(\mathrm{kg} / \mathrm{m}^{2}\right)$, atrial fibrillation (Minnesota Codes 8-3-1 or 8-3-2), ST-T changes (Minnesota 4-1 to 4-3 or 5-1 to 5-3), ethanol intake (never-drinkers, ex-drinkers, or current drinkers), serum cholesterol (mmol/L), and diabetes mellitus (a fasting glucose level of $>7.0 \mathrm{mmol} / \mathrm{l}$, a non-fasting glucose level of $>11.1$ $\mathrm{mmol} / \mathrm{l}$, or the use of medication for diabetes).

\section{Statistical Analysis}

The paired Student's t-test was used to compare the values of baseline potential confounding factors and smoking status between incident cases and control subjects. The chi-square test was used to compare proportions of cases and control subjects. The odds ratios of total and subtypes of dementia were estimated according to the smoking status, tertiles of smoking years and tertiles of pack-years of smoking using the conditional logistic regression model. Adjustments for systolic blood pressure (mmHg), use of antihypertensive medication (yes vs. no), BMI $\left(\mathrm{kg} / \mathrm{m}^{2}\right)$, alcohol intake (non-current drinkers vs. current drinkers), atrial fibrillation (yes vs. no), ST-T abnormalities (yes vs. no), serum total cholesterol (mmol/L), and diabetes mellitus (yes vs. no) were applied for multivariable analysis. A trend across the categories for smoking years and pack-years of smoking was conducted by assigning median values for each category. To examine effect modification, we conducted stratified analyses according to sex and use of antihypertensive medication (yes and no), and checked statistical interactions 
by using cross-product terms of smoking status and stratifying variables. The presence of sex interaction for the associations between smoking and risk of dementia was tested using cross-product terms of smoking status and sex variables. All p-values for statistical tests were two-tailed and values $<0.05$ were considered to reflect significant difference. All analyses were conducted using the SAS statistical package version 8.2 (SAS Institute Inc., Cary, NC). 


\section{RESULTS}

During 2000 to 2004, we identified 208 dementia cases, comprising 95 dementia cases with history of stroke and 113 dementia cases without such history.

Table 1 shows the risk characteristics of dementia cases and control subjects. The average age at the time of a given an annual cardiovascular survey and physical examination during 1981 to 1994 was 69 years for total and subtypes of dementia, and 68-69 years for controls. The proportion of men was $36 \%$ for total dementia, $43 \%$ for dementia with history of stroke, $30 \%$ for without such history, and 30 to $43 \%$ for controls. The proportions of current smokers were higher for total dementia for controls. The prevalence of hypertension, diabetes, and ST-T abnormalities was higher for total and subtypes of dementia than for controls. These case-control differences were more evident for dementia with history of stroke than for dementia without such history.

Table 2 shows age-, sex-, and survey-year-matched and multivariable adjusted odds ratios $(95 \% \mathrm{CI})$ for total and subtypes of dementia according to smoking status. The multivariable odds ratios for current versus never-smokers were 2.3 (1.1-4.7) for total dementia, 2.4 (0.8-7.7) for dementia with history of stroke and 2.3 (0.9-6.0) for dementia without it. There was no significant excess risk among past smokers. We further analyzed the data by stratifying current smokers into two categories; $<20$ and $\geq 20$ cigarettes/day and found a dose-response relationship for total dementia.

We also analyzed the data by stratifying past and current smokers into three categories according to the number of years of and the pack-years of smoking, and found a dose-response relationship with the number of years of smoking and risk of total dementia (p for trend $=0.04$ ), but not with the pack-years of smoking ( $\mathrm{p}$ for trend $=0.21$ ) (Table3). The 
dose-response relationship with the number of years of smoking was more pronounced for dementia with history of stroke although the excess risk did not reach statistical significance. We examined for potential effect modification by stratifying the analyses for sex and use of antihypertesive medication (yes and no) (data not shown). There were no statistically significant interactions between smoking status and sex ( $\mathrm{p}$ for interaction=0.43) or use of antihypertesive medication ( $\mathrm{p}$ for interaction=0.80) for total dementia. 


\section{DISCUSSION}

Our prospective study was the first to show that current smoking was associated with a two-fold increase in the risk of disabling dementia among Japanese. The association between smoking and risk of disabling dementia did not vary between persons with and without history of stroke. The Pooled European Studies of Dementia reported that current smokers had an increased risk of total dementia (relative risk $[R R]=1.4 ; 95 \%$ confidence interval $[C I]=1.0-1.9)$ and Alzheimer's disease (RR=1.7; 95\% CI=1.2-2.5) compared with individuals who had never smoked. ${ }^{9}$ Another prospective study of mixed ethnic groups (African-Americans, whites and Hispanics) in the USA reported that current smokers had an increased risk of Alzheimer's disease ( $\mathrm{RR}=1.7$; 95\% $\mathrm{CI}=1.1-2.8)$ compared with those who had never smoked. ${ }^{10}$ The Honolulu-Asia Ageing Study reported that current smokers had an increased risk of vascular dementia (odds ratio $[\mathrm{OR}]=1.7 ; 95 \% \mathrm{CI}=1.0-2.9$ ), but not for Alzheimer's disease, compared with those who had never smoked; however, this association was no longer significant after adjustment for cardiovascular and respiratory factors. ${ }^{11}$

A previous prospective study in Japan showed an association between smoking and increased risk of mortality from dementia. ${ }^{21}$ That study followed 265,118 individuals aged $\geq 40$ years over a period of 17 years, and documented 69 deaths from senile dementia with cerebrovascular lesions and 120 deaths from senile dementia without cerebrovascular lesions. The age-adjusted RR of senile dementia without cerebrovascular lesions for current smokers compared with never smokers was $1.6(90 \% \mathrm{CI}=1.1-2.4)$, but no association was found between dementia with cerebrovascular lesions. However, that study did not adjust for potential confounding variables including blood pressure levels, and the diagnosis of dementia was not validated because the diagnosis relied only on death certificates. 
A previous study found that the higher pack-year exposure was associated with greater cognitive decline among current and former smokers. ${ }^{22}$ We found no significant association between the pack-year exposure and risk of total dementia, either dementia with or without history of stroke. However, we found an association between the number of years of smoking and the risk of total dementia, which may suggest that a long-term exposure rather than a high dose of smoking may increase the risk.

Several mechanisms could account for the adverse effect of smoking on the risk of vascular dementia. Smoking causes arterio-atherosclerosis by directly injuring endothelial cells, ${ }^{23}$ it reduces high-density lipoprotein cholesterol levels, ${ }^{24}$ and it accelerates thrombus formation by increasing plasma fibrinogen ${ }^{25}$ and platelet aggregability ${ }^{26}$, decreasing fibrinolytic activities, ${ }^{27}$ increasing hematocrit ${ }^{28}$ and reducing blood flow in the brain due to vasoconstriction. $^{29}$

Considering the association between smoking and Alzheimer's disease, a previous case-reference study showed that patients with this disorder and a history of smoking had lower regional cerebral blood flow in the temporal, parietal, occipital and frontal lobes bilaterally, compared with individuals who had never smoked. ${ }^{30}$ When blood flow reaches a critical threshold of cerebral hypoperfusion, brain capillaries can be regionally damaged, and the normal release of nitric oxide and glucose, as well as oxygen delivery to the neurons, can be impaired..$^{31}$ These conditions can lead to cerebral metabolic dysfunction, which might contribute to the progression of neuronal degeneration. ${ }^{32}$ Increased oxidative stress caused by smoking $^{33,34}$ is another potential cause of Alzheimer's disease through enhanced neurotoxcity. ${ }^{35}$

Nicotine, which is a major component of tobacco smoke, has a neuroprotective effect via a reduction of amyloid aggregation and neurotoxicity. ${ }^{36}$ However, any potential beneficial 
effect of nicotine is likely to be overwhelmed by the adverse effects of the other components of tobacco smoke. ${ }^{33}$

The strengths of our current study were its prospective design, and the comprehensive nature of cardiovascular surveys, which allowed that the association between smoking and risk of dementia is adjusted for important potential confounding variables. Moreover, because we used annual cardiovascular surveys that were carried out $\geq 5$ years before the endpoint determination, severe dementia was unlikely to be present at the time of the risk-factor assessment. This would enhance our confidence that smoking behaviour was not influenced by subclinical dementia.

The major limitations of our study were as follows. First, there might have been a residual confounding effect on the association between smoking and risk of dementia. Although we adjusted for selected cardiovascular risk, we could not exclude the possible influence of unmeasured risk factors such as education level ${ }^{37}$ and marital status. ${ }^{38}$ Second, the misclassification for diagnosis of dementia was inevitable. The study had low sensitivity but high specificity for the diagnosis of disabling dementia. Thus, cases of disabling dementia were less likely to be contaminated by false-positive cases, but the false negative cases were likely to exist in the control pool. However, due to the low prevalence of disabling dementia (approximately $5-6 \%$ of persons aged $\geq 65$ ), the contamination by false positive cases in the controls may be small. The controls could be contaminated by patients with mild cognitive impairments in the present study, which may attenuate the associations. Third, the smoking exposure could be misclassified due to self-reporting. However, previous studies showed reported that self-reported smoking was fairly reliable. ${ }^{39,40}$ In the present study, we examined answer-reanswer reliability with 1-year interval between 1981 and 1994 and found that the reliability for self-reported smoking status was high; $97 \%$ for never-smokers, $87 \%$ for past smokers, 93\% for current smokers. Fourth, the smoking-dementia associations did not reach 
statistical significance when we examined two subtypes of dementia, probably due to the low statistical power to detect the effects. Fifth, there was a long duration between the time at smoking status surveyed and the time at dementia diagnosed, i.e., 6 to 21 years, which would make interpretation for the results difficult. However, the results did not change materially when the analyses were conducted, stratified by the time duration 6-14 years $(n=461)$ and $15-21$ years $(n=163)$. For example, odds ratios $(95 \% \mathrm{CI})$ of total dementia in relation to current smokers were $1.8(0.8-4.2)$ in the time duration of 6 -14 years, and 2.0 (0.5-7.6) in the time duration of 15-21 years. There were no statistical interactions of two periods with smoking status, duration or the amount of smoking. Finally, we did not have imaging studies to confirm the subtypes of dementia, and dementia without a history of stroke did not necessarily correspond to Alzheimer's disease. However, the proportion of Alzheimer's disease among persons with dementia without history of stroke was 79\% in a previous Japanese study, ${ }^{41}$ suggesting that dementia without history of stroke in present study are likely to be Alzheimer's disease. In contrast, only $2 \%$ of persons with history of stroke were Alzheimer's disease in that study, suggesting that Alzheimer's disease cases were less likely to exist in cases of dementia with history of stoke.

In conclusion, our prospective study showed that smoking was associated with an increased risk of disabling dementia. The smoking-dementia association did not vary by stroke history. A dose-response relationship was observed between the duration of smoking and the risk, and a two-fold excess risk was found for smokers of $\geq 45$ years in duration. Long-term cigarette smoking may raise the risk of disabling dementia. 


\section{ACKNOWLEDGMENTS}

This study was supported in part by a Grant-in-Aid for exploratory research from the Japan Society for Promotion of Science (no. 17659183 in 2005-2006). The authors thank Dr. Fumio Yamashita, University of Tsukuba, Yoko Wakabayashi, Sawako Toriumi, Kumiko Shimada, Mieko Inagawa, and Miyuki Oshima, Kyowa Municipal Office (presently Chikusei Municipal Office), for the technical support.All persons mentioned have seen and approved mention of their names in the article. 


\section{REFERENCES}

1. Aevarsson O, Skoog I. A population-based study on the incidence of dementia disorders between 85 and 88 years of age. J Am Geriatr Soc. 1996; 44:1455-60.

2. Roman RC. Vascular dementia prevention: a risk factor analysis. Cerebrovasc Dis 2005; 20: 91-100.

3. Kivipelto M, Helkala EL, Laakso MP, Hanninen T, Hallikainen M, Alhainen K, Iivonen S, Mannermaa A, Tuomilehto J, Nissinen A, Soininen H. Apolipoprotein E epsilon4 allele, elevated midlife total cholesterol level, and high midlife systolic blood pressure are independent risk factors for late-life Alzheimer disease. Ann Intern Med 2002; 137: 149-55.

4. Skoog I, Lernfelt B, Landahl S, Palmertz B, Andreasson LA, Nilsson L, Persson G, Oden A, Svanborg A. 15-year longitudinal study of blood pressure and dementia. Lancet 1996; 347: 1141-5.

5. Whitmer RA, Sidney S, Selby J, Johnston SC, Yaffe K. Midlife cardiovascular risk factors and risk of dementia in late life. Neurology 2005; 64: 277-81.

6. Almeida OP, Hulse GK, Lawrence D, Flicker L. Smoking as a risk factor for Alzheimer’s disease: contrasting evidence from a systematic review of case-control and cohort studies. Addiction 2001; 97: 15-28.

7. Riggs JE. Smoking and Alzheimer's disease: protective effect or differential survival bias? Lancet 1993; 342: 793-4.

8. Doll R, Peto R, Wheatley K, Gray R, Sutherland I. Mortality in relation to smoking: 40 years’ observations on male British doctors. Br Med J 1994; 309: 901-911.

9. Launer LJ, Andersen K, Dewey ME, Letenneur L, Ott A, Amaducci LA, Brayne C, Copeland JR, Dartigues JF, Kragh-Sorensen P, Lobo A, Martinez-Lage JM, Stijnen T, 
Hofman A. Rates and risk factors for dementia and Alzheimer's disease. Results from EURODEM pooled analyses. Neurology 1999; 52: 78-84.

10. Merchant C, Tang MX, Albert S, Manly J, Stern Y, Mayeux R. The influence of smoking on the risk of Alzheimer’s disease. Neurology 1999; 52: 1408-12.

11. Tyas SL, White LR, Petrovitch H. Mid-life smoking and late-life dementia: the Honolulu-Asia Aging Study. Neurobiol Aging 2003; 24: 589-96.

12. Iso H, Moriyama Y, Sato S, Kitamura A, Tanigawa T, Yamagishi K, Imano H, Ohira T, Okamura T, Naito Y, Shimamoto T. Serum total homocysteine concentrations and risk of stroke and its subtypes in Japan. Circulation 2004; 109: 2766-72.

13. Yamagishi K, Iso H, Kitamura A, Sankai T, Tanigawa T, Naito Y, Sato S, Imano H, Ohira T, Shimamoto T. Smoking raises the risk of total and ischemic strokes in hypertensive men. Hypertens Res 2003; 26: 209-17.

14. Campbell JC, Ikegami N. Long-term care insurance comes to Japan. Health Aff 2000; 19: 26-39.

15. Matsuda S, Yamamoto M. Long-term care insurance and integrated care for the aged in Japan. Int J Integr Care 2001; 1: e28.

16. Health and Welfare Bureau for the Elderly of Japan. Standardized assessment manual for the levels of Activity Daily Living deficiencies among elderly with dementia. 1993. (in Japanese).

17. Levy R. Aging-associated cognitive decline. Int Psychogeriatr 1994; 6: 63-8.

18. Iso H, Rexrode K, Hennekens CH, Manson JE. Application of computer tomography-oriented criteria for stroke subtype classification in a prospective study. Ann Epidemiol 2000; 10: 81-87. 
19. Shimamoto T, Komachi Y, Inada H, Doi M, Iso H, Sato S, Kitamura A, Iida M, Konishi M, Nakanishi N, Terao A, Naito Y, Kojima S. Trends for coronary heart disease and stroke and their risk factors in Japan. Circulation 1989; 79: 503-515.

20. Rothman KJ, Greenland S. Modern Epidemiology Second Eds. Philadelphia, PA: Lippincott Williams \& Wilkins, 1998:93-99.

21. Hirayama T. Large cohort study on the relation between cigarette smoking and senile dementia without cerebrovascular lesions. Tob Control 1992; 1; 176-9.

22. Ott A, Andersen K, Dewey ME, Letenneur L, Brayne C, Copeland JR, Dartigues JF, Kragh-Sorensen P, Lobo A, Martinez-Lage JM, Stijnen T, Hofman A, Launer LJ; EURODEM Incidence Research Group. Effect of smoking on global cognitive function in nondemented elderly. Neurology 2004; 62: 920-4.

23. Rogers RL, Meyer JS, Judd BW, Mortel KF. Abstention from cigarette smoking improves cerebral perfusion among elderly chronic smokers. JAMA 1985; 235: 2970-4.

24. Nagy J, Demaster EG, Wittmann I, Shultz P, Raij L. Induction of endothelial injury by cigarette smoking. Endothelium 1997; 5: 251-63.

25. Meade TW, Imeson J, Stirling Y. Effects of changes in smoking and other characteristics on clotting factors and the risk of ischaemic heart disease. Lancet 1987; 2: 986-8.

26. Pittilo RM, Clarke JM, Harris D, Mackie IJ, Rowles PM, Machin SJ, Woolf N. Cigarette smoking and platelet adhesion. Br J Haematol 1984; 58: 627-32.

27. Newby DE, Wright RA, Labinjoh C, Ludlam CA, Fox KA, Boon NA, Webb DJ. Endothelial dysfunction, impaired endogenous fibrinolysis, and cigarette smoking: a mechanism for arterial thrombosis and myocardial infarction. Circulation 1999; 99: 1411-5. 
28. Whisnant JP, Homer D, Ingall TJ, Baker HL Jr, O'Fallon WM, Wievers DO. Duration of cigarette smoking is the strongest predictor of severe extracranial carotid artery atherosclerosis. Stroke 1990; 21: 707-14.

29. Smith JR, Landau SA. Smokers’ polycythemia. N Engl J Med 1978; 298: 6-10.

30. Siennicki-Lantz A, Lilja B, Elmstahl S. Cerebral perfusion deficits in age-associated memory impairment. The role of tobacco smoking. Aging Clin Exp Res 2002; 14: 108-16.

31. de la Torre JC, Stefano GB. Evidence that Alzheimer's disease is a microvascular disorder: the role of constitutive nitric oxide. Brain Res Brain Res Rev 2000; 34:119-36.

32. de la Torre JC. Hemodynamic consequences of deformed microvessels in the brain in Alzheimer's disease. Ann N Y Acad Sci 1997; 26: 75-91.

33. U.S Department of Health and Human Services. Reducing the health consequences of smoking: 25 years of progress. Rockville, MD: U.S. Department of Health and Human Services, Public Health Services, Centers for Disease Control, Center for Chronic Disease Prevention and Health Promotion, Office on Smoking and Health (DHHS Publication No. [CDC] 89-8411); 1989.

34. Traber MG, van der Vliet A, Reznick AZ, Cross CE. Tobacco-related diseases. Is there a role for antioxidant micronutrient supplementation? Clin Chest Med 2000; 21:173-87.

35. Butterfield DA, Drake J, Pocernich C, Castegna A. Evidence of oxidative damage in Alzheimer's disease brain: central role for amyloid beta-peptide. Trends Mol Med 2001; 7:548-54.

36. Zamani MR, Allen YS. Nicotine and its interaction with beta-amyloid protein: a short review. Biol Psychiatry 2001; 49: 221-32.

37. Yip AG, Brayne C, Matthews FE. Risk factors for incident dementia in England and Wales: The Medical Research Council Cognitive Function and Ageing Study. A population-based nested case-control study. Age Ageing 2006; 35:154-160. 
38. Helmer C, Damon D, Letenneur L, Fabrigoule C, Barberger-Gateau P, Lafont S, Fuhrer R, Antonucci T, Commenges D, Orgogozo JM, Dartigues JF. Marital status and risk of Alzheimer's disease: a French population-based cohort study. Neurology 1999; 53:1953-1958.

39. Vartiainen E, Seppala T, Lillsunde P, Puska P.Validation of self reported smoking by serum cotinine measurement in a community-based study. J Epidemiol Community Health 2002; 56:167-170.

40. Wagenknecht LE, Burke GL, Perkins LL, Haley NJ, Friedman GD. Misclassification of smoking status in the CARDIA study: a comparison of self-report with serum cotinine levels. Am J Public Health 1992; 82: 33-36.

41. Yoshitake T, Kiyohara Y, Kato I, Ohmura T, Iwamoto H, Nakayama K, Ohmori S, Nomiyama K, Kawano H, Ueda K, Sueishi K, Tsuneyoshi M, Fujishima M. Incidence and risk factors of vascular dementia and Alzheimer’s disease in a defined elderly Japanese population: the Hisayama Study. Neurology 1995; 45: 1161-68. 


\section{Table 1.}

Baseline characteristics of study population by case and control. 
Table 2.

Odds ratios for dementia according to cigarette smoking status. 


\section{Table 3.}

Odds ratios for dementia according to years of smoking and pack-years of smoking. 
Table 1

\begin{tabular}{|c|c|c|c|c|c|c|c|c|c|c|c|c|}
\hline & & Age & Men & $\begin{array}{l}\text { Systolic } \\
\text { blood } \\
\text { pressure }\end{array}$ & $\begin{array}{c}\text { Use of } \\
\text { antihypertensive } \\
\text { medication }\end{array}$ & BMI & $\begin{array}{c}\text { Alcohol } \\
\text { Intake }\end{array}$ & $\begin{array}{l}\text { Current } \\
\text { Smokers }\end{array}$ & $\begin{array}{c}\text { Serum } \\
\text { Cholesterol }\end{array}$ & Diabetes & $\begin{array}{c}\text { Atrial } \\
\text { fibrillation }\end{array}$ & $\begin{array}{c}\text { ST-T } \\
\text { abnormalities }\end{array}$ \\
\hline & $\mathrm{n}$ & $\mathrm{y}$ & $\%$ & $\mathrm{mmHg}$ & $\%$ & $\mathrm{~kg} / \mathrm{m} 2$ & $\%$ & $\%$ & $\mathrm{mmol} / \mathrm{L}$ & $\%$ & $\%$ & $\%$ \\
\hline \multicolumn{13}{|l|}{ Total demenita } \\
\hline cases & 208 & 69.1 & 35.6 & $139.3 *$ & 34.0 & 23.3 & 24.0 & $22.6 *$ & 5.33 & 9.8 & 1.9 & 9.6 \\
\hline control subjects & 416 & 68.6 & 35.6 & 135.6 & 35.0 & 23.7 & 22.0 & 16.1 & 5.26 & 6.7 & 2.4 & 9.1 \\
\hline \multicolumn{13}{|c|}{ Dementia with history of stroke } \\
\hline cases & 95 & 69.4 & 42.1 & $142.2 *$ & 42.1 & 23.3 & 28.4 & 25.3 & 5.31 & $13.8 *$ & 3.2 & $14.7 * *$ \\
\hline control subjects & 190 & 68.9 & 42.1 & 134.4 & 37.4 & 23.6 & 27.4 & 17.4 & 5.19 & 6.0 & 3.7 & 7.9 \\
\hline \multicolumn{13}{|c|}{ Dementia without history of stroke } \\
\hline cases & 113 & 68.9 & 30.1 & 137.0 & 27.4 & 23.2 & 20.2 & 20.4 & 5.34 & 6.6 & 0.9 & 5.4 \\
\hline control subjects & 226 & 68.4 & 30.1 & 136.6 & 32.7 & 23.9 & 17.5 & 15.0 & 5.32 & 7.2 & 1.3 & 10.2 \\
\hline
\end{tabular}

$* p<0.05$ and ${ }^{* *} p<0.10$ (difference between cases and controls) 
Table 2

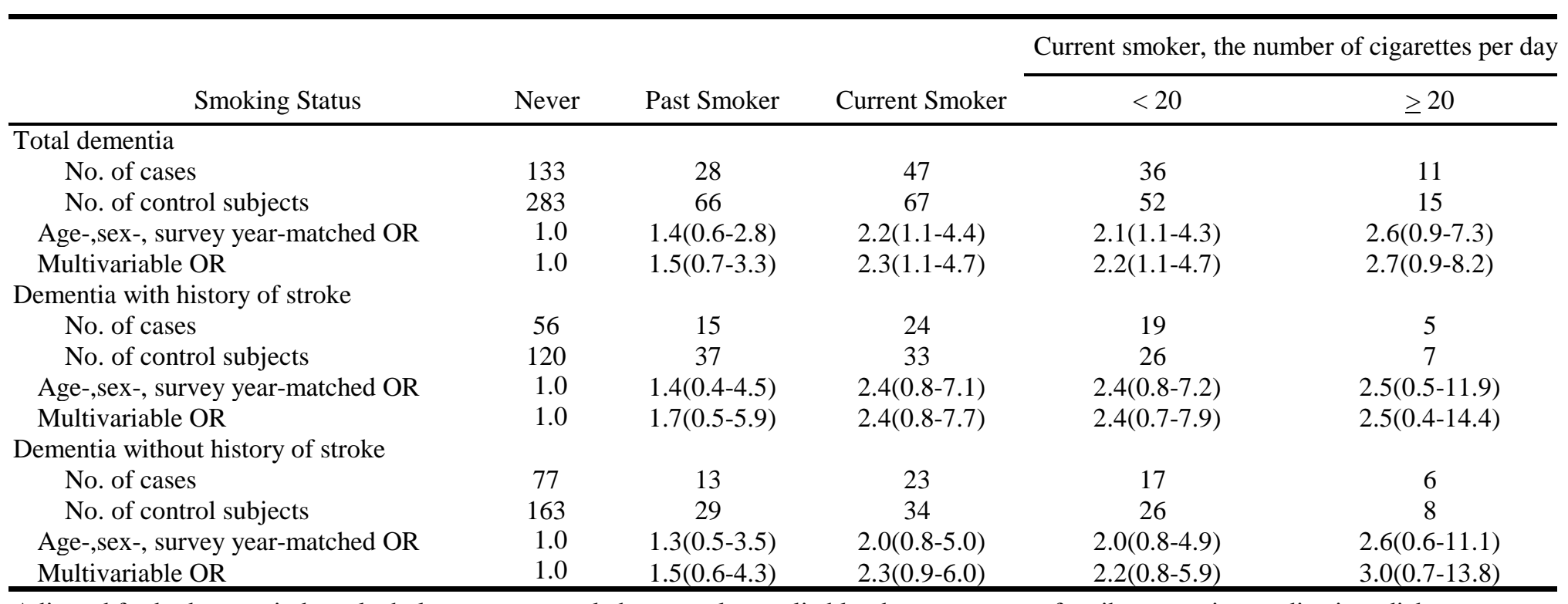

Adjusted for body mass index, alcohol use, serum total choresterol, systolic blood pressure, use of antihypertensive medication, diabetes mellitus, atrial fibrillation and ST-T abnormality. 
Table 3

\begin{tabular}{|c|c|c|c|c|c|}
\hline \multirow[b]{2}{*}{ Smoking Status } & \multirow[b]{2}{*}{ Never } & \multicolumn{3}{|c|}{ Years of smoking } & \multirow[t]{2}{*}{$P$ for trend } \\
\hline & & $0<$ years $<34$ & $34<$ years $<45$ & years $>45$ & \\
\hline \multicolumn{6}{|l|}{ Total demenita } \\
\hline No. of cases & 133 & 23 & 22 & 30 & \\
\hline No. of control subjects & 283 & 45 & 43 & 45 & \\
\hline Age-,sex-, survey year-matched OR & 1.0 & $1.5(0.7-3.2)$ & $1.8(0.8-4.3)$ & $2.3(1.1-5.2)$ & 0.04 \\
\hline Multivariable OR & 1.0 & $1.7(0.8-3.7)$ & $2.0(0.8-5.2)$ & $2.3(1.0-5.4)$ & 0.04 \\
\hline \multicolumn{6}{|l|}{ Dementia with history of stroke } \\
\hline No. of cases & 56 & 8 & 13 & 18 & \\
\hline No. of control subjects & 120 & 21 & 25 & 24 & \\
\hline Age-,sex-, survey year-matched OR & 1.0 & $1.3(0.4-4.2)$ & $2.4(0.6-9.5)$ & 3.4(1.0-12.1) & 0.05 \\
\hline Multivariable OR & 1.0 & $1.7(0.5-6.0)$ & $2.7(0.5-13.2)$ & $3.0(0.7-12.3)$ & 0.13 \\
\hline \multicolumn{6}{|l|}{ Dementia without history of stroke } \\
\hline No. of cases & 77 & 15 & 9 & 12 & \\
\hline No. of control subjects & 163 & 24 & 18 & 21 & \\
\hline Age-,sex-, survey year-matched OR & 1.0 & $1.8(0.7-4.6)$ & $1.5(0.5-4.9)$ & $1.7(0.6-4.9)$ & 0.29 \\
\hline \multirow[t]{2}{*}{ Multivariable OR } & 1.0 & $2.0(0.7-5.7)$ & $1.8(0.5-6.1)$ & $1.8(0.6-5.7)$ & 0.24 \\
\hline & & \multicolumn{3}{|c|}{ Pack-years of smoking } & $P$ for trend \\
\hline Smoking Status & Never & $0<$ years $<28$ & $28 \leq$ years $<46$ & years $\geq 46$ & \\
\hline \multicolumn{6}{|l|}{ Total demenita } \\
\hline No. of cases & 133 & 31 & 18 & 26 & \\
\hline No. of control subjects & 283 & 47 & 42 & 44 & \\
\hline Age-,sex-, survey year-matched OR & 1.0 & $1.9(1.0-3.8)$ & $1.4(0.6-3.2)$ & $2.0(0.8-4.5)$ & 0.23 \\
\hline Multivariable OR & 1.0 & $2.1(1.0-4.3)$ & $1.3(0.5-3.3)$ & $2.1(0.9-5.1)$ & 0.21 \\
\hline \multicolumn{6}{|l|}{ Dementia with history of stroke } \\
\hline No. of cases & 56 & 15 & 10 & 14 & \\
\hline No. of control subjects & 120 & 25 & 21 & 24 & \\
\hline Age-,sex-, survey year-matched OR & 1.0 & $2.0(0.7-6.1)$ & $1.7(0.5-6.2)$ & $2.2(0.6-7.5)$ & 0.36 \\
\hline Multivariable OR & 1.0 & $2.5(0.8-8.1)$ & $1.3(0.3-5.3)$ & $2.2(0.5-8.5)$ & 0.57 \\
\hline \multicolumn{6}{|l|}{ Dementia without history of stroke } \\
\hline No. of cases & 77 & 16 & 8 & 12 & \\
\hline No. of control subjects & 163 & 22 & 21 & 20 & \\
\hline Age-,sex-, survey year-matched OR & 1.0 & $1.9(0.8-4.6)$ & $1.1(0.3-3.6)$ & $1.7(0.5-5.8)$ & 0.43 \\
\hline Multivariable OR & 1.0 & $2.1(0.8-5.5)$ & $1.0(0.3-3.8)$ & $2.1(0.6-7.3)$ & 0.33 \\
\hline
\end{tabular}

Adjusted for body mass index, alcohol use, serum total choresterol, systolic blood pressure, use of antihypertensive medication, diabetes mellitus, atrial fibrillation and ST-T abnormality. 